\title{
Comparison of PD-L1 Scores in Primary Kidney Tumors Versus Accompanying Venous Tumor Thrombi: Retrospective, Comparative, Monocentric Study in Treatment-Naive Patients
}

\author{
Mehrdad Mazdak · Rieke Ringlstetter - Pouriya Faraj Tabrizi • \\ Meryem Akkoyun · Mathias Wolters · Jessica Schmitz · Jan Hinrich Bräsen • \\ Inga Peters · Markus Antonius Kuczyk · Hossein Tezval
}

Received: January 21, 2021 / Accepted: April 1, 2021 / Published online: May 21, 2021

(C) The Author(s) 2021

\begin{abstract}
Introduction: Renal cell carcinoma (RCC), an immunogenic tumor, is the most common form of kidney cancer worldwide. Immune checkpoint inhibitors (ICIs) play an important role in the treatment of metastatic RCC. Programmed death-ligand (PD-L1) has already been proposed as a possible prognosticator for ICIs effectiveness. To elucidate the feasible role of ICIs in neoadjuvant settings, we have assessed the most common PD-L1 expression modalities [tumor proportion score (TPS), combined positivity score (CPS) and inflammatory cell (IC) score] in primary tumors (PTs) and venous tumor thrombi (VTT) in first diagnosed, previously untreated RCC patients with accompanying VTT.
\end{abstract}

M. Mazdak · R. Ringlstetter · P. F. Tabrizi ·

M. Akkoyun · M. Wolters - I. Peters .

M. A. Kuczyk · H. Tezval (ه)

Department of Urology and Urological Oncology,

Hanover Medical School (MHH), Carl-Neuberg-

Straße 1, 30625 Hanover, Germany

e-mail: Tezval.Hossein@mh-hannover.de

\section{R. Ringlstetter}

Department of Information and Communication, Faculty III, University of Applied Sciences and Arts Hanover, 30539 Hanover, Germany

J. Schmitz · J. H. Bräsen

Department of Pathology, Hanover Medical School (MHH), 30625 Hanover, Germany
Methods: Between January 1999 and December 2016, 71 patients with a first diagnosed, untreated, locally advanced RCC (aRCC) ( $\geq$ pT3a) underwent surgery in Hanover Medical School (MHH). PD-L1 expression was examined separately in PTs and VTT using the CPS, IC score and TPS. We also considered the age at the time of the initial surgery and gender as probable influencing factors. By using a cutoff value of 1 (1\%), PD-L1 expression levels in PTs and VTT were assessed to enable the determination of any frequency differences.

Results: Positive scores for PTs were shown by 54 (CPS), 53 (IC score) and 34 (TPS) patients, whereas in VTT, positive scores were evaluated for a total of 50 (CPS), 47 (IC-score) and 36 (TPS) patients. No statistically significant differences were obtained between the PD-L1 expression immunoscores for PTs and VTT. The covariates age at the time of the initial surgery and gender could not be statistically proven to influence the differences in PD-L1 expression between the VTT and PTs.

Conclusion: To the best of our knowledge, this research is the largest study to investigate PD-L1 expression in PTs and VTT in 71 cases. It could have relevance for the future development of neoadjuvant immunotherapy options, particularly in aRCC with VTT.

Keywords: Renal cell carcinoma; Immune checkpoint inhibitors; Programmed-death ligand 1; Venous tumor thrombi 


\section{Key Summary Points}

\section{Background}

RCC is the primary kidney cancer worldwide. Despite knowing different improved available operative techniques as well as various systemic therapies, management of aRCC with accompanying venous tumor thrombi (VTT) or metastatic renal cell carcinoma (mRCC) is still challenging. The role of immunotherapies in mRCC is growing and well established. Programmed deathligand 1 (PD-L1) expression, as a response prognosticator of immunotherapies, has been suggested in previous oncology studies

\section{What did the study ask?}

In this study, we assess three varied PD-L1 expression modalities in primary RCC and accompanying VTT pathology specimens to achieve more insight into their use in these scenarios and compare them to determine possible differences

\section{Outcomes/conclusions}

Showing a similar PD-L1 expression pattern in 71 primary locally advanced kidney tumors and concomitant VTT in the largest study so far, we show the potential role of immunotherapies as a neoadjuvant therapy concept in aRCC with accompanying VTT

As an alternative curative option by downstaging the tumor compared with the primary operation, the most advantageous administration form of immunotherapies in aRCC demands further molecular research as well as comprehensive clinical trials

\section{DIGITAL FEATURES}

This article is published with digital features, including a summary slide, to facilitate understanding of the article. To view digital features for this article go to https://doi.org/10.6084/ m9.figshare.14340323.

\section{INTRODUCTION}

Renal cell carcinoma (RCC), defined as a group of malignancies, is one of the most common neoplasms arising in the kidney and has different subtypes and distinctive features [1, 2]. RCC, as an immunogenic malignancy, has some unique manifestations, such as the development of venous tumor thrombi (VTT) with cephalad extensions during progression in $5-10 \%$ of cases $[3,4]$. Approximately $30 \%$ of all RCCs are diagnosed as synchronous or metachronous metastatic disease [5].

Since the introduction of immune checkpoint inhibitors (ICIs), the sequencing therapies used for metastatic renal cell carcinoma (mRCC) have undergone significant modification. ICIs produce fewer side effects compared to previous targeted therapeutic agents. They improve both health-related quality of life (HRQoL) and clinical outcomes [6, 7]. Nevertheless, not all patients benefit from their use [8], but due to a lack of predictive biomarkers it is still unclear which patients respond best/worse to this treatment.

The main ICI targets are cytotoxic T-lymphocyte-associated protein 4 (CTLA-4) and programmed cell death protein 1 (PD-1), as two immune checkpoint receptors, as well as programmed death-ligand 1 (PD-L1), as an immune checkpoint protein $[8,9]$.

PD-L1 expression analysis with immunohistochemistry (IHC) is an established biomarker by which patients are divided into potential responders and non-responders [8]. Different scoring methods for PD-L1 staining have been developed. The combined positivity score (CPS), inflammatory cell (IC) score and tumor proportion score (TPS) are the most frequently mentioned [10]. However, a distinctive, concurrent measurement of all three of 
these parameters, as well as a comparison among them, is still lacking in mRCC and locally advanced RCC (aRCC) scenarios. It is still unclear whether PD-L1 status is a suitable predictor of a response to ICIs. To date, there have been conflicting results regarding the use of PD-L1 status [5].

Using knowledge of PD-L1 expression in different parts of each tumor [8], and with the objective of improving our understanding of the implications and effectiveness of ICIs in aRCC patients, our study aims to assess and compare PD-L1 expression using the CPS, IC score and TPS in 71 primary RCC tumors (PTs) and VTT, including 57 with vena cava involvement. This will enable us to elucidate the immunologic characteristics of PTs and VTT, as well as the rational role played by preoperative adjuvant immunotherapy, aimed at downstaging, in aRCC with VTT scenarios.

\section{METHODS}

\section{Patient Characteristics}

Our retrospective study includes 71 patients with de novo preoperative clinically diagnosed and postoperative pathologically confirmed $\geq$ pT3a RCC (per 7th TNM edition [11]) with an accompanying VTT (Table 1). Because of their tumors, all patients underwent radical nephrectomy at the Department of Urology and Urological Oncology at the Hanover Medical School between January 1999 and December 2016. No patient had received any previous preoperative systemic therapy, such as tyrosine kinase inhibitors (TKIs) or ICIs, or any other form of intervention. The study was approved by the ethics committee of the Hanover Medical School (MHH) (ethics votes number 1696-2013). All procedures performed in our study were in accordance with the ethical standards of the institutional research committee and with the 1964 Helsinki Declaration and its later amendments or comparable ethical standards.
Table 1 Patient characteristics

\begin{tabular}{|c|c|c|}
\hline \multirow[t]{2}{*}{ Characteristics } & \multicolumn{2}{|c|}{ Total $(n=71)$} \\
\hline & $\begin{array}{l}\text { No. of } \\
\text { patients }\end{array}$ & $\%$ \\
\hline \multicolumn{3}{|l|}{ Gender } \\
\hline Male & 42 & 59.2 \\
\hline Female & 29 & 40.8 \\
\hline $\begin{array}{l}\text { Median age at the time of the initial } \\
\text { surgery in years (range) }\end{array}$ & $67(41-87)$ & \\
\hline \multicolumn{3}{|l|}{ Side of the kidney } \\
\hline Left & 25 & 35.2 \\
\hline Right & 46 & 64.8 \\
\hline \multicolumn{3}{|l|}{ Tumor type } \\
\hline Clear-cell RCC (ccRCC) & 61 & 85.9 \\
\hline Papillary RCC (pRCC) & 7 & 9.9 \\
\hline Chromophobe RCC & 1 & 1.4 \\
\hline Sarcomatoid RCC & 2 & 2.8 \\
\hline \multicolumn{3}{|l|}{ Grading (Fuhrman) } \\
\hline G1 & 1 & 1.4 \\
\hline G2 & 31 & 43.7 \\
\hline G3 & 36 & 50.7 \\
\hline G3/G4 & 1 & 1.4 \\
\hline G4 & 2 & 2.8 \\
\hline \multicolumn{3}{|l|}{ T stage* } \\
\hline T3a & 14 & 19.7 \\
\hline $\mathrm{T} 3 \mathrm{~b}$ & 52 & 73.2 \\
\hline $\mathrm{T} 3 \mathrm{c}$ & 4 & 5.6 \\
\hline $\mathrm{T} 4$ & 1 & 1.4 \\
\hline \multicolumn{3}{|l|}{ N stage* } \\
\hline $\mathrm{ND}^{* *}$ & 36 & 50.7 \\
\hline 0 & 19 & 26.8 \\
\hline 1 & 11 & 15.5 \\
\hline 2 & 5 & 7.0 \\
\hline \multicolumn{3}{|l|}{ M stage* } \\
\hline $\mathrm{ND}^{* *}$ & 44 & 62.0 \\
\hline
\end{tabular}


Table 1 continued

\begin{tabular}{llc}
\hline Characteristics & \multicolumn{2}{l}{ Total $(n=71)$} \\
\cline { 2 - 3 } & $\begin{array}{l}\text { No. of } \\
\text { patients }\end{array}$ & $\%$ \\
\hline 0 & 4 & 5.6 \\
1 & 23 & 32.4 \\
\hline
\end{tabular}

${ }^{*}$ Note: The tumor classification is based on the seventh edition of the TNM staging system of the American Joint Commission on Cancer/Union for International Cancer Control (AJCC/UICC)

${ }^{* *}$ Not determined

\section{Tissue Samples, Staining and Scoring}

Pathologic tumor assessment and PD-L1 stainings were carried out in cooperation with the Institute of Pathology, MHH, Germany. Selected samples included the worst differentiation and the rim of necrosis for the primary tumor and cava thrombus, if possible. Immunostaining of $3-5 \mu \mathrm{m}$ formaldehyde-fixed, paraffinembedded tissue sections was routinely performed on an automated platform (Ventana ULTRA, Ventana Medical Systems, Tucson, AZ, USA) using a PD-L1 antibody (clone 22C3, 1:40, Agilent, Santa Clara, CA, USA) after heat-induced epitope retrieval with CC1 solution (Ventana Medical Systems). Positive controls (tonsil tissue) were included in all staining procedures.

The evaluation of the following scores was carried out by a trained pathologist, Dr. Jan Hinrich Bräsen (JHB). These scores have been comprehensively described in the literature $[10,12]$.

$$
\begin{gathered}
\text { IC - score }(\%)=\frac{\text { Stained immune cells }}{\text { Tumor area }} \\
\text { TPS }(\%)=\frac{\text { Number of stained tumor cells }}{\text { Total number of tumor cells }}
\end{gathered}
$$

\section{Statistical Methods}

Statistical evaluations were carried out using the Statistical Analysis System (SAS) statistical software package, University Edition, version 9.4 SAS Institute Inc., Cary, NC, USA (https://www. sas.com/en_us/software/sas9.html). By utilizing SAS software, the quantitative characteristics of the samples were described using the PROC MEANS procedure to evaluate the sample size, mean, median, standard deviation, and minimum and maximum values. Graphical representation of the results was performed using the PROC SGPLOT procedure and additions such as HISTOGRAM for the creation of histograms and $V B O X$ for the creation of box plots. To compare the three PD-L1 scores being analyzed (CPS, IC score and TPS) in PTs and VTT, a two-sample $t$ test for dependent samples was used. If a twosample $t$-test for dependent samples was not applicable, a Wilcoxon signed-rank test was performed. To compare the PD-L1 scoring characteristics of thrombi and primary tumors in different age categories, we applied a KruskalWallis with $S$-test as the global test. A twosample $t$-test for independent samples was used to assess the role that gender plays in the different PD-L1 scores. A Mann-Whitney $U$ test or Welch's unequal variances $t$-test were used if a two-sample $t$-test for independent samples was not appropriate. Comparison of the frequencies of PD-L1-positive $(\geq 1 / \geq 1 \%)$ and -negative $(<1 /<1 \%)$ PTs and VTT was performed using a McNemar test. A $p$ value $<0.05$ (two-sided) was

$$
\begin{aligned}
\mathrm{CPS}= & \frac{\text { Number of stained tumor cells }+ \text { Number of stained mononuclear inflammatory cells }}{\text { Total number of tumor cells }}
\end{aligned}
$$


considered statistically significant, unless otherwise mentioned.

\section{RESULTS}

The samples obtained from our 71 patients were examined between November 2019 and June 2020 to determine PD-L1 expression status through the CPS, IC score and TPS modalities in PT and VTT samples. To determine the

Table 2 PD-L1-positive and -negative lesions according to their CPS

\begin{tabular}{llllll}
\hline $\begin{array}{l}\text { Primary } \\
\text { tumors }\end{array}$ & $\begin{array}{l}\text { Venous tumor } \\
\text { thrombi }\end{array}$ & Total & $\%$ & $\boldsymbol{p}$ value \\
\cline { 2 - 4 } & Negative & Positive & & & \\
\hline CPS & & & & & \\
Negative & 8 & 9 & 17 & 23.9 & 0.3938 \\
Positive & 13 & 41 & 54 & 76.1 & \\
Total & 21 & 50 & 71 & 100.0 & \\
\hline
\end{tabular}

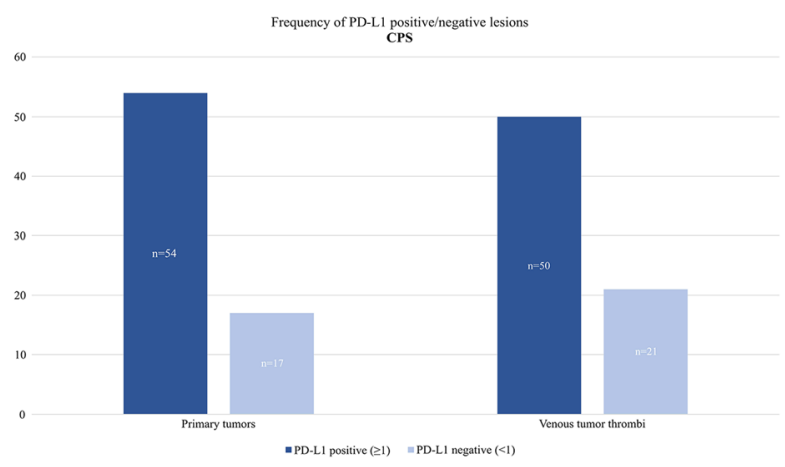

Fig. 1 Frequency of PD-L1 positive $(\geq 1)$ and negative $(<1)$ lesions according to their CPS. CPS Combined Positive Score influence of other variables, the characteristics of age and gender were also evaluated.

\section{CPS Analysis}

A total of 17 PTs had a CPS of $<1(23.9 \%)$. Meanwhile, 21 VTT had a CPS of $<1(29.6 \%)$. A total of 54 PTs (76.1\%) and 50 VTT (70.4\%) had a CPS of $\geq 1$. Descriptively, the differences were minimal (Table 2, Fig. 1). Both PTs and VTT have more PD-L1-positive specimens than negative ones. In a total of 13 cases, the PTs had a positive CPS $(\geq 1)$, while the associated VTT had a negative CPS $(<1)$. Concurrently, in nine cases, the VTT had a positive CPS, while the associated PTs were negative using the McNemar test ( $p=0.3938)$ (Table 2).

The mean CPS of the VTT is 6.65 score points (median $=3.00 ; \mathrm{SD}=8.16$ ), slightly higher than the mean CPS (6.27 score points) of the PTs $($ median $=4.00, \mathrm{SD}=8.49)(p=0.7533,95 \% \mathrm{CI}$ $[-2.0230 ; 2.7836])$. Scores range from 0.00 to 55.00 score points in the PTs and from 0.00 to 40.00 score points in the VTT (Table 3, Fig. 2).

\section{IC Score Analysis}

A total of 18 PTs had an IC score of $<1 \%$ $(25.4 \%) ; 24$ VTT had an IC score of $<1 \%$ (33.8\%). A total of 53 PTs (74.6\%) and 47 VTT $(66.2 \%)$ had an IC-score of $\geq 1 \%$ (Table 4 , Fig. 3). Again, there were more PD-L1-positive specimens than negative ones. By using a McNemar test, in 13 cases the PTs were evaluated as having a positive IC score, while the associated VTT were evaluated as having a negative IC score. In seven cases, the VTT had positive scores, while the PTs had negative scores $(p=0.1797)$ (Table 4$)$.

Table 3 Evaluation of CPS values according to tumor type

\begin{tabular}{lcccccrr}
\hline Lesion & $\boldsymbol{N}$ & Mean & Median & SD & Min & Max & $\boldsymbol{p}$ value \\
\hline PT & 71 & 6.27 & 4.00 & 8.49 & 0.00 & 55.00 & - \\
VTT & 71 & 6.65 & 3.00 & 8.16 & 0.00 & 40.00 & - \\
Difference between VTT and PT & 71 & 0.38 & 0.00 & 10.15 & -51.00 & 25.00 & 0.7533 \\
\hline
\end{tabular}



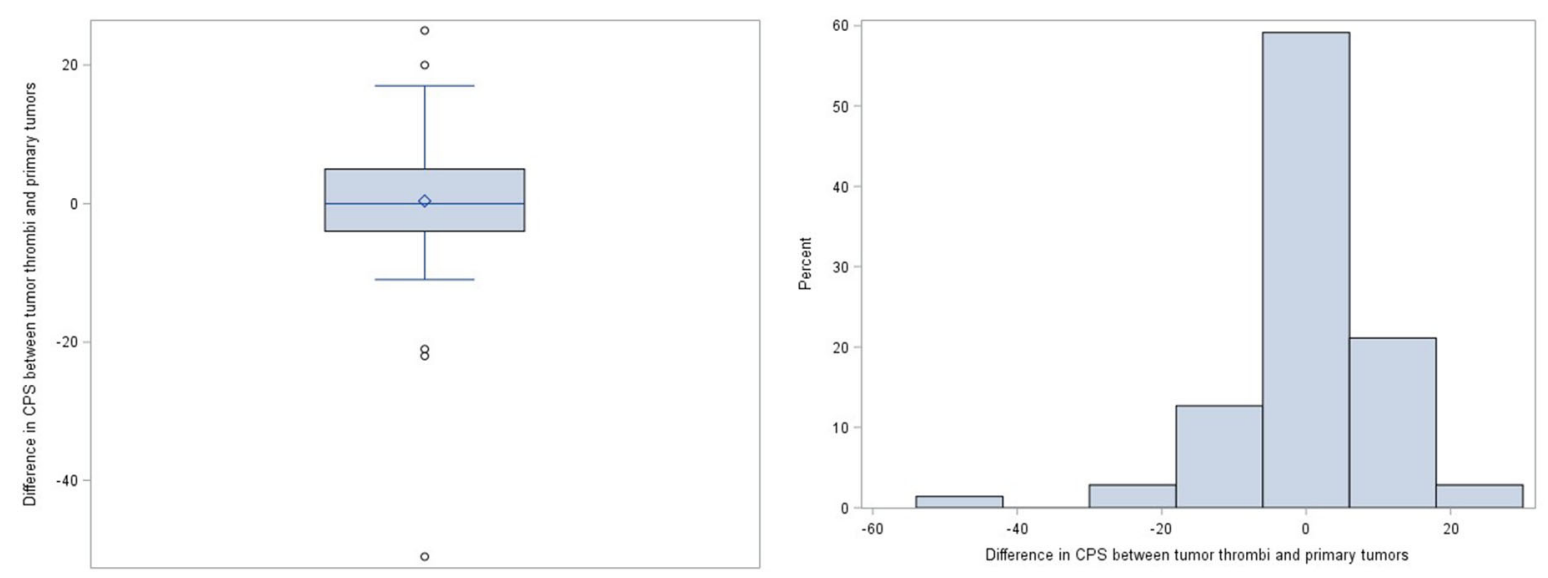

Fig. 2 Box plot and histogram illustrating the difference in CPS between venous tumor thrombi and primary tumors. CPS Combined Positive Score

Table 4 PD-L1-positive and -negative lesions according to their IC score

\begin{tabular}{llccll}
\hline $\begin{array}{l}\text { Primary } \\
\text { tumors }\end{array}$ & $\begin{array}{l}\text { Venous tumor } \\
\text { thrombi }\end{array}$ & Total & & $\boldsymbol{p}$ value \\
\cline { 2 - 3 } & Negative & Positive & & & \\
\hline IC score & & 7 & 18 & 25.4 & 0.1797 \\
Negative & 11 & 40 & 53 & 74.6 & \\
Positive & 13 & 47 & 71 & 100.0 & \\
Total & 24 & & & & \\
\hline
\end{tabular}

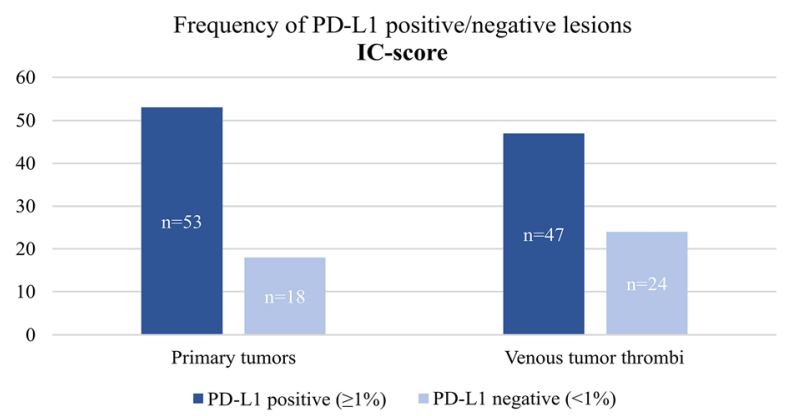

Fig. 3 Frequency of PD-L1 positive ( $\geq 1 \%$ ) and negative $(<1 \%)$ lesions according to their IC-Score. IC-Score Inflammatory Cell-Score

The mean IC score of the VTT was $4.06 \%$, marginally higher than the IC score $(3.82 \%)$ of the PTs $\quad($ median $=2.00 \% ; \quad \mathrm{SD}=4.35 \%)$ $(p=0.7303,95 \%$ CI $[-1.1404 ; 1.6193])$. In the PTs, scores ranged from 0.00 to $20.00 \%$, while in the VTT scores ranged from 0.00 to $30.00 \%$ (Table 5, Fig. 4).

\section{TPS Analysis}

A total of 37 PTs had a TPS of $<1 \%(52.1 \%)$. On the other hand, 35 VTT had a TPS of $<1 \%$ (49.3\%). A total of 34 PTs (47.9\%) and 36 VTT $(50.7 \%$ ) had a TPS of $\geq 1 \%$ (Table 6, Fig. 5). Descriptively, the differences were minimal. In the PTs, there were slightly more PD-L1-negative specimens than positive ones. In the VTT, the number of PD-L1-positive specimens exceeded the number of negative specimens by only one. In comparison, the results of a McNemar test showed that 11 PTs were positive $(\geq 1 \%)$, while the associated VTT were negative $(<1 \%)$. In 13 cases, the VTT were positive, while the associated PTs were negative $(p=0.6831)$ (Table 6).

The mean TPS of the VTT was $2.41 \%$, a value which was higher than the mean TPS $(1.94 \%)$ of the PTs $\quad($ median $=0.00 \% ; \quad \mathrm{SD}=5.43 \%$ ) ( $p=0.2490$ ). Scores ranged from 0.00 to $40.00 \%$ in the PTs, while in the VTT scores ranged from 0.00 to $15.00 \%$. (Table 7 , Fig. 6). The box plot (Fig. 6) illustrates that there are some statistical outliers, and to take these into account, a Wilcoxon signed-rank test was performed. 
Table 5 Evaluation of IC scores according to tumor type

\begin{tabular}{lllllrll}
\hline Lesion & $\boldsymbol{N}$ & Mean (\%) & Median (\%) & \multicolumn{1}{l}{ SD (\%) } & Min (\%) & Max (\%) & $\boldsymbol{p}$ value \\
\hline PT & 71 & 3.82 & 2.00 & 4.35 & 0.00 & 20.00 & - \\
VTT & 71 & 4.06 & 2.00 & 5.62 & 0.00 & 30.00 & - \\
Difference between VTT and PT & 71 & 0.24 & 0.00 & 5.83 & -15.00 & 20.00 & 0.7303 \\
\hline
\end{tabular}
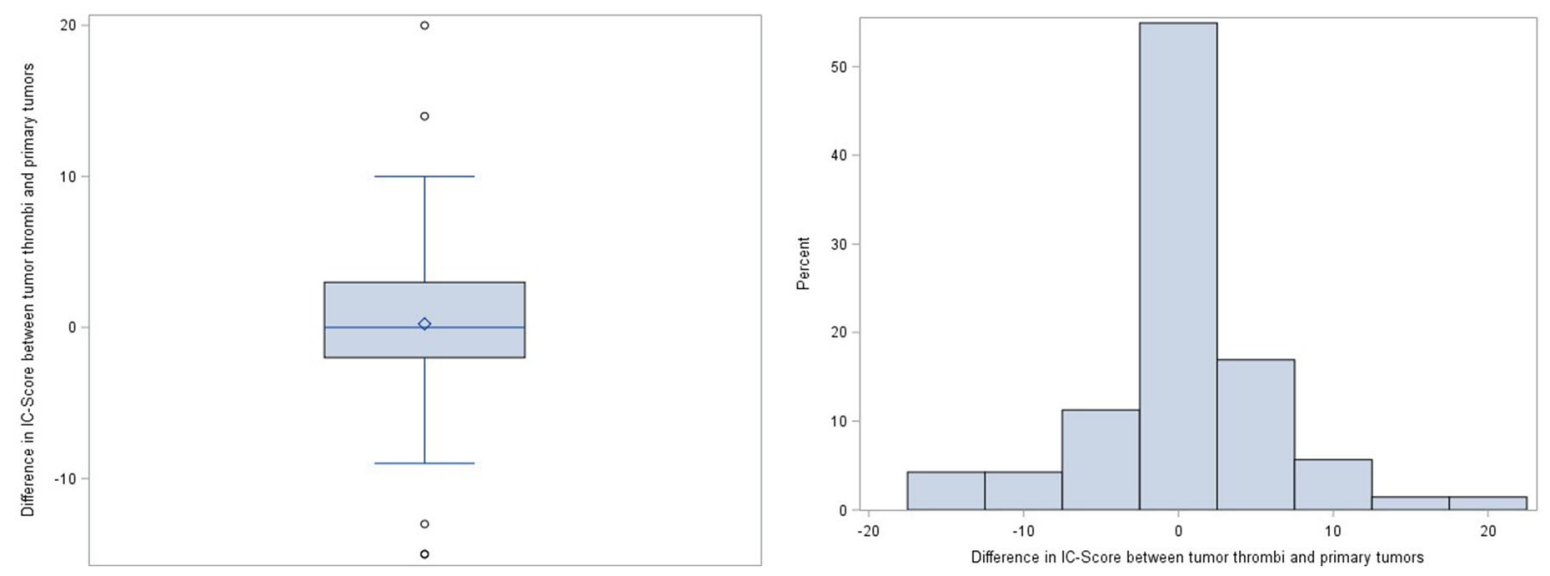

Fig. 4 Box plot and histogram illustrating the difference in IC-Score between venous tumor thrombi and primary tumors. IC-Score Inflammatory Cell-Score

Table 6 PD-L1 positive and negative lesions according to their TPS

\begin{tabular}{lllllll}
\hline $\begin{array}{l}\text { Primary } \\
\text { tumors }\end{array}$ & $\begin{array}{l}\text { Venous tumor } \\
\text { thrombi }\end{array}$ & & Total & $\%$ & $\begin{array}{l}\boldsymbol{p} \\
\text { value }\end{array}$ \\
\cline { 2 - 4 } & Negative & Positive & & & & \\
\hline TPS & & & & & & \\
Negative & 24 & 13 & 37 & 52.1 & 0.6831 \\
Positive & 11 & 23 & 34 & 47.9 & \\
Total & 35 & 36 & 71 & 100.0 & \\
\hline
\end{tabular}

\section{Correlation Between Age at the Time of the Initial Surgery and PD-L1 Levels}

Hereafter, the age of the patient at the time of his/her initial surgery will be analyzed as a factor that could potentially influence PD-L1 expression, by considering the CPS, IC score and TPS.

The mean differences between VTT and PTs in the three age categories were relatively

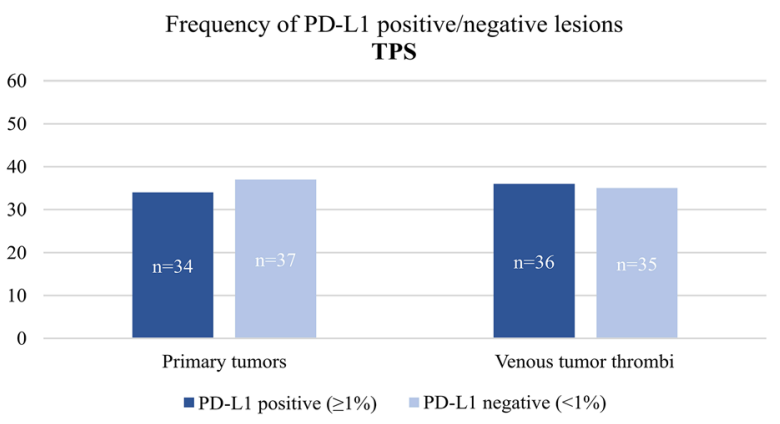

Fig. 5 Frequency of PD-L1 positive $(\geq 1 \%)$ and negative $(<1 \%)$ lesions according to their TPS. TPS Tumor Proportion Score

similar in all PD-L1 modalities (Table 8). The small differences that exist between the age categories can also be seen in the following box plots (Figs. 7, 8, and 9). Negative differences mean that PD-L1 expression is higher in the PTs, while positive differences mean that PD-L1 expression is higher in the VTT.

Since the requirements for a one-way analysis of variance (ANOVA) are not met in the case 
Table 7 Evaluation of TPS values according to tumor type

\begin{tabular}{lllllcll}
\hline Lesion & $\boldsymbol{N}$ & Mean (\%) & Median (\%) & SD (\%) & Min (\%) & Max (\%) & $\boldsymbol{p}$ value \\
\hline PT & 71 & 1.94 & 0.00 & 5.43 & 0.00 & 40.00 & - \\
VTT & 71 & 2.41 & 1.00 & 4.25 & 0.00 & 15.00 & - \\
Difference between VTT and PT & 71 & 0.46 & 0.00 & 5.97 & -35.00 & 15.00 & 0.2490 \\
\hline
\end{tabular}
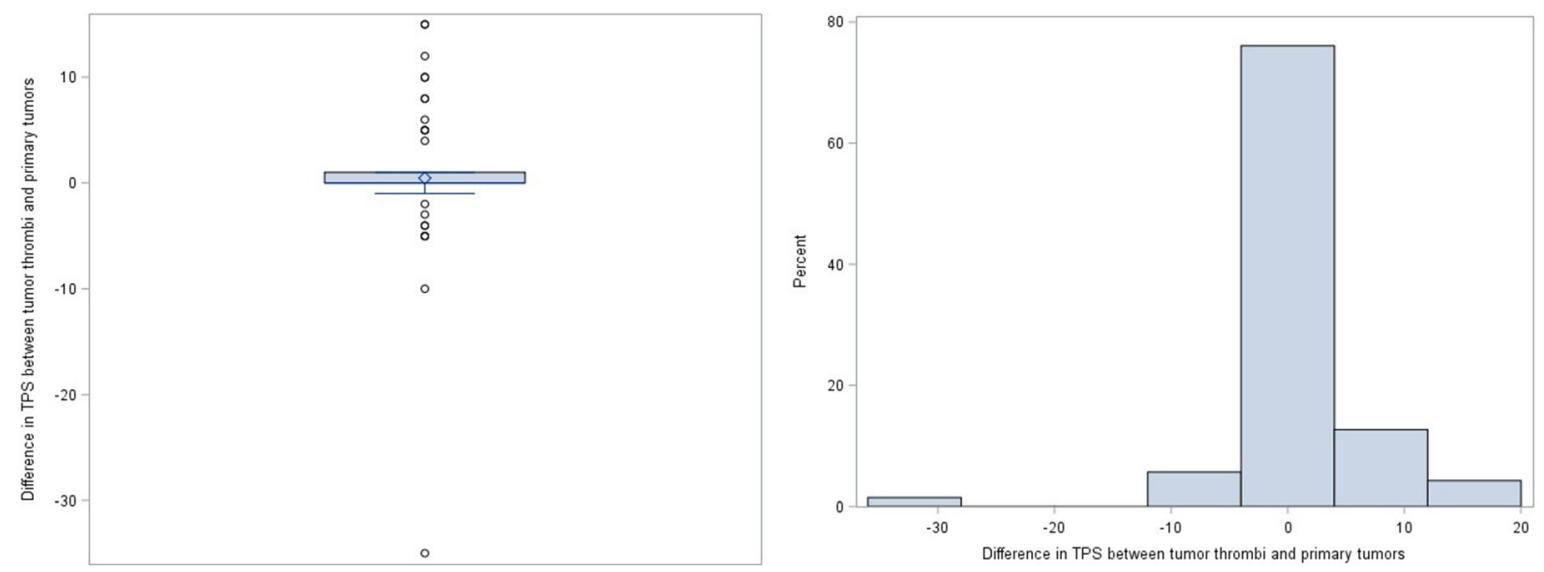

Fig. 6 Box plot and histogram illustrating the difference in TPS between venous tumor thrombi and primary tumors. TPS Tumor Proportion Score

Table 8 Evaluation of PD-L1 levels according to the age of the patient at the time of the initial surgery

\begin{tabular}{lrrrrrrr}
\hline $\begin{array}{l}\text { Age category (in years) } \\
\text { (Difference between VTT and PT) }\end{array}$ & $\boldsymbol{N}$ & Mean & Median & SD & Min & Max & $\boldsymbol{p}$ value \\
\hline CPS & & & & & & & \\
$1(<60)$ & 18 & 0.94 & 0.50 & 9.31 & -22.00 & 17.00 & 0.5168 \\
$2(\geq 60$ to $<70)$ & 26 & -0.54 & 0.00 & 5.43 & -11.00 & 11.00 & - \\
$3(\geq 70)$ & 27 & 0.89 & 0.00 & 13.83 & -51.00 & 25.00 & - \\
IC score $(\%)$ & & & & & & & \\
$1(<60)$ & 18 & -0.56 & 0.00 & 5.15 & -15.00 & 7.00 & 0.5915 \\
$2(\geq 60$ to $<70)$ & 26 & -0.81 & 0.00 & 4.89 & -15.00 & 8.00 & - \\
$3(\geq 70)$ & 27 & 1.78 & 0.00 & 6.87 & -13.00 & 20.00 & - \\
TPS $(\%)$ & & & & & & & \\
$1(<60)$ & 18 & 1.44 & 0.00 & 5.10 & -5.00 & 15.00 & 0.3581 \\
$2(\geq 60$ to $<70)$ & 26 & 0.62 & 0.00 & 3.14 & -5.00 & 10.00 & - \\
$3(\geq 70)$ & 27 & -0.33 & 0.00 & 3.14 & -35.00 & 12.00 & - \\
\hline
\end{tabular}




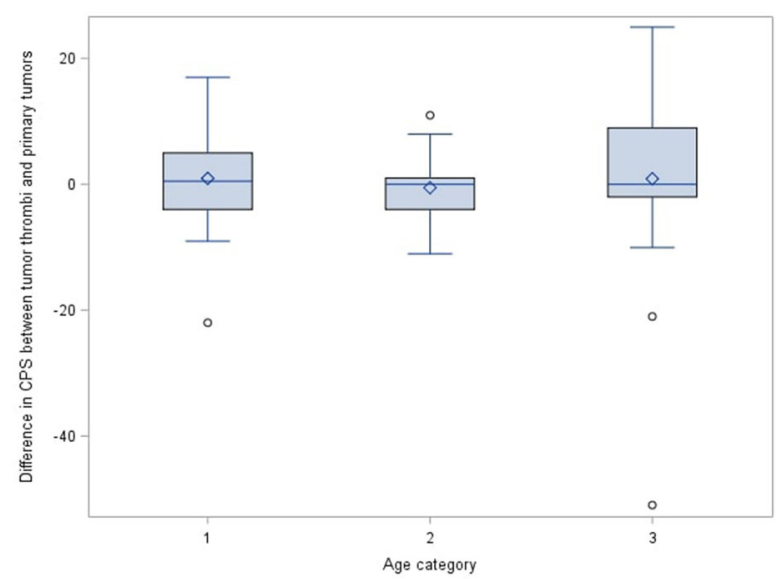

Fig. 7 Box plots of the difference in CPS between venous tumor thrombi and primary tumors in the three age categories. $1=<60$ years of age, $2=\geq 60$ to $<70$ years of age, $3=\geq 70$ years of age. CPS Combined Positive Score

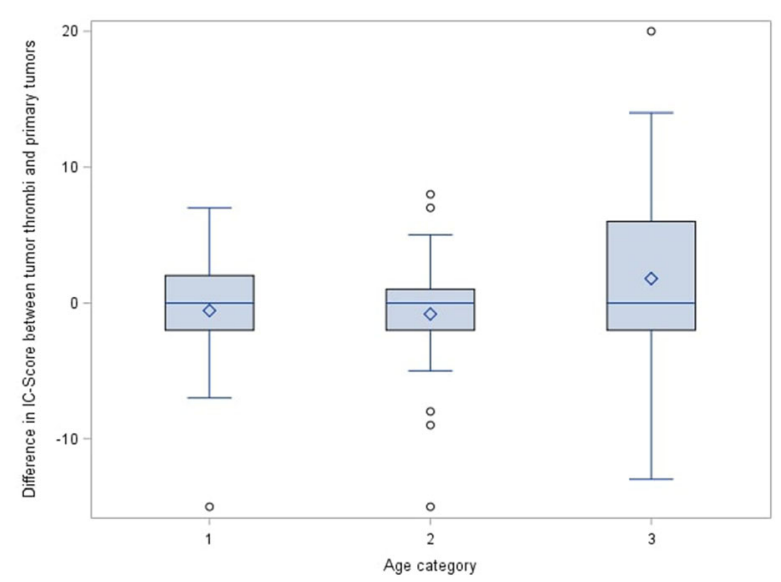

Fig. 8 Box plots of the difference in IC-Score between venous tumor thrombi and primary tumors in the three age categories. $1=<60$ years of age, $2=\geq 60$ to $<70$ years of age, $3=\geq 70$ years of age. IC-Score Inflammatory Cell-Score

of data with heterogeneous variance or data where normal distribution cannot be assumed, the homogeneity of variance of the data was checked using Levene's test and normal distribution is examined using the KolmogorovSmirnov test with a significance level of $p<0.10$ (two sided). The $p$ values of Levene's test obtained for the CPS $(p=0.2294)$, IC score $(p=0.3343)$ and TPS $(p=0.3587)$ were not statistically significant, and therefore the variances could be assumed to be homogeneous. Still,

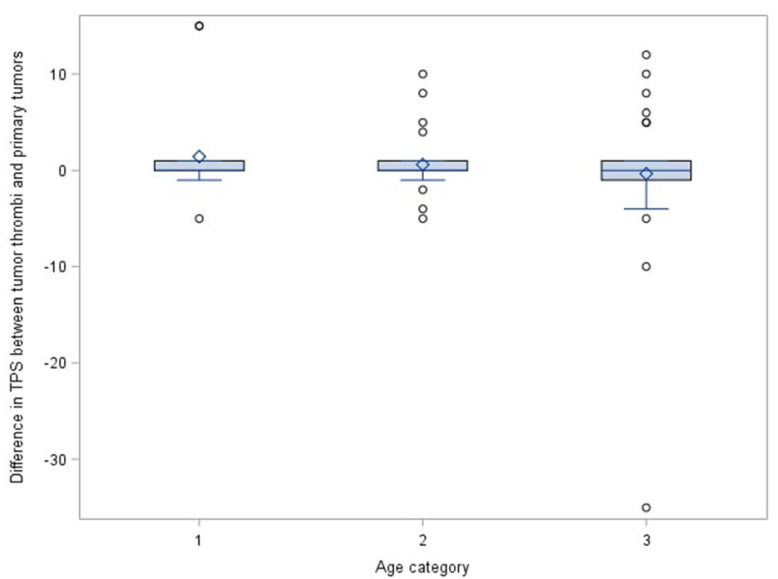

Fig. 9 Box plots of the difference in TPS between venous tumor thrombi and primary tumors in the three age categories. $1=<60$ years of age, $2=\geq 60$ to $<70$ years of age, $3=\geq 70$ years of age. TPS Tumor Proportion Score

some of the $p$ values of the Kolmogorov-Smirnov test for the CPS (age $<60[p>0.1500]$; age $\geq 60$ to $<70 \quad[p=0.0909] ; \quad$ age $\geq 70$ $[p<0.0100])$, IC score (age $<60[p=0.0357]$; age $\geq 60 \quad$ to $<70 \quad[p<0.0100]) ; \quad$ age $\geq 70$ $[p=0.0839])$ and TPS (age $<60[p<0.0100]$; age $\geq 60$ to $<70 \quad[p<0.0100], \quad$ age $\geq 70$ $[p<0.0100])$ were significant, which is why the non-parametric Kruskal-Wallis test was performed instead of the parametric one-way ANOVA. Thus, the Kruskal-Wallis test was carried out to compare the mean differences between VTT and PTs for the three PD-L1 modalities in the three age categories.

For the Kruskal-Wallis H-test, a $p$ value $\leq 0.20$ (two-sided) was considered statistically significant. Regarding CPS, patients $<60$ years of age had the highest mean difference between VTT and PTs with 0.94 score points $\quad($ median $=0.50, \quad \mathrm{SD}=9.31)$, while patients $\geq 60$ to $<70$ years of age had a mean difference of -0.54 score points (median $=0.00, \mathrm{SD}=5.43$ ) and patients $\geq 70$ years of age had a mean difference of 0.89 score points (median $=0.00, \mathrm{SD}=13.83)(p=0.5168)$ (Table 8). Since the global $p$ value of the $S$-test for CPS was not significant, at least two age categories could not be statistically proven to differ in terms of the mean difference between their VTT and PTs. 
In terms of IC-score, patients $\geq 70$ years of age had the highest mean difference $(1.78 \%)$ between VTT and PTs (median $=0.00 \%, \mathrm{SD}=$ $6.87 \%$ ), patients $\geq 60$ to $<70$ years of age had a mean difference of $-0.81 \%$ (median $=0.00 \%$, $\mathrm{SD}=4.89 \%)$ and patients $<60$ years of age had a mean difference of $-0.56 \%$ (median $=0.00 \%$, $\mathrm{SD}=5.15 \%)(p=0.5915)$ (Table 8$)$. The global $p$ value of the $H$-test was not significant, and therefore there was no statistical difference between at least two age categories for the IC score.

Regarding TPS, patients $<60$ years of age had the highest mean difference (1.44\%) between VTT and PTs (median $=0.00, \mathrm{SD}=$ $5.10 \%$ ), patients $\geq 60$ to $<70$ years of age had a mean difference of $0.62 \%$ (median $=0.00 \%$, $\mathrm{SD}=3.14)$ and patients $\geq 70$ years of age had a mean difference of $-0.33 \%$ (median $=0.00 \%$, $\mathrm{SD}=8.26 \%)(p=0.3581)$ (Table 8$)$. Again, the global $p$ value of the $H$-test was not significant, and therefore no statistical difference between VTT and PTs in the three age categories could be found. As all the global Kruskal-Wallis $H$-tests were not statistically significant, no further pairwise tests were performed.

\section{Correlation Between Gender and PD-L1 Levels}

In all PD-L1 modalities (CPS, IC score and TPS), female patients had marginally higher mean differences than male patients. These mean differences were positive in value, indicating that the mean PD-L1 expression of the VTT was higher than the PD-L1 expression of the PTs. In the case of male patients, the mean differences were negative in the case of the CPS and IC score, which indicates that the mean PD-L1 expression of the PTs is higher than the mean PD-L1 expression of the VTT. Male patients only had a positive mean difference in the case of TPS (Table 9). Variation in the mean differences was only marginal in all PD-L1 modalities, as illustrated in the following box plots and histograms (Figs. 10, 11 and 12).

In the case of CPS, since the standard deviations showed considerable variation (Table 9), Welch's unequal variances $t$-test was selected for statistical testing. The difference in CPS between VTT and PTs for female patients (mean $=1.83, \mathrm{SD}=6.48)$ was slightly higher than that for male patients (mean $=-0.62$, $\mathrm{SD}=12.04) \quad(p=0.2728,95 \%$ CI $\quad[-1.9713$; 6.8645]).

In relation to the IC score, the standard deviations were rather similar (Table 9), and therefore a two-sample $t$-test for independent samples was selected for statistical testing. For female patients, the difference in IC score was slightly higher $($ mean $=1.52 \%, \quad S D=5.03 \%)$ than the corresponding value for male patients $($ mean $=-0.64 \%, \quad S D=6.23 \%) \quad(p=0.1257$, $95 \%$ CI $[-0.6201 ; 4.9403])$.

Table 9 Evaluation of the PD-L1 levels according to the gender of the patient

\begin{tabular}{lrrrrrrr}
\hline Gender (difference between VTT and PT) & $\boldsymbol{N}$ & Mean & Median & SD & Min & Max & $\boldsymbol{p}$ value \\
\hline CPS & & & & & & & \\
Female & 29 & 1.83 & 1.00 & 6.48 & -10.00 & 16.00 & 0.2728 \\
$\quad$ Male & 42 & -0.62 & 0.00 & 12.04 & -51.00 & 25.00 & - \\
IC score (\%) & & & & & & & \\
$\quad$ Female & 29 & 1.52 & 0.00 & 5.03 & -15.00 & 10.00 & 0.1257 \\
Male & 42 & -0.64 & -0.50 & 6.23 & -15.00 & 20.00 & - \\
TPS (\%) & & & & & & & \\
Female & 29 & 0.90 & 0.00 & 4.11 & -10.00 & 15.00 & 0.3581 \\
Male & 42 & 0.17 & 0.00 & 7.01 & -35.00 & 15.00 & - \\
\hline
\end{tabular}



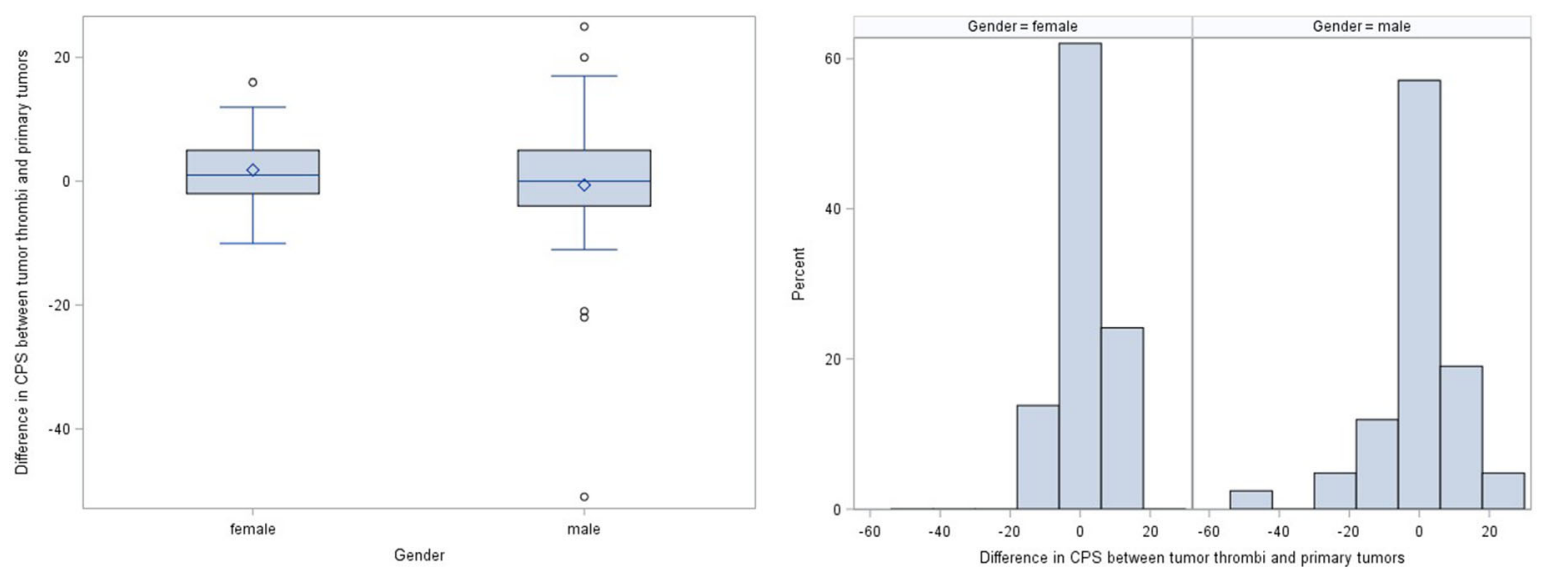

Fig. 10 Box plots and histograms of the difference in CPS between venous tumor thrombi and primary tumors according to gender. CPS Combined Positive Score
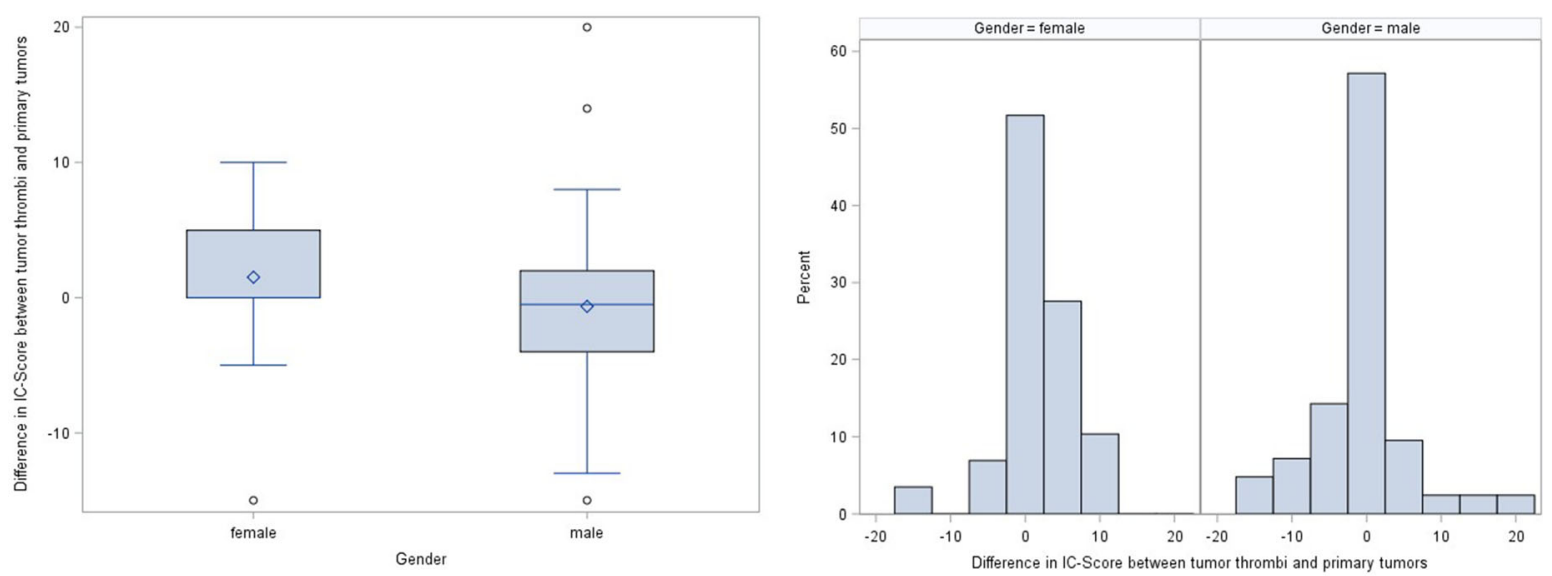

Fig. 11 Box plots and histograms of the difference in IC-Score between venous tumor thrombi and primary tumors according to gender. IC-Score Inflammatory Cell-Score

The TPS data showed some statistical outliers (Fig. 12), and therefore a Mann-Whitney- $U$ test was performed. Again, there was a greater difference between the TPS of VTT and PTs in female patients (mean $=0.90 \%, \quad S D=4.11 \%$ ) than in male patients (mean $=0.17 \%, \mathrm{SD}=$ 7.01\%) $(p=0.3581)$.

\section{DISCUSSION}

Recent studies have advanced the hypothesis that PD-L1 expression is an influential predictive biomarker, and one that can play a conclusive role in immunotherapies [8]. The exact dynamic that exists between ICIs and PD-L1 expression is still not properly understood. By establishing the range of PD-L1 expression in different parts of each tumor, the central aim of this work is to gain further knowledge about PD-L1 expression in both primary RCC tumors and their accompanying venous tumor thrombi.

The sequencing of systemic therapies as a recommendation for the treatment of advanced and metastatic RCC has, over time, has undergone considerable evolution. Primarily, cytokine therapy with interleukin-2 (IL-2) and interferon-alfa (IFNa) have been reported as immunotherapies with relatively positive and 

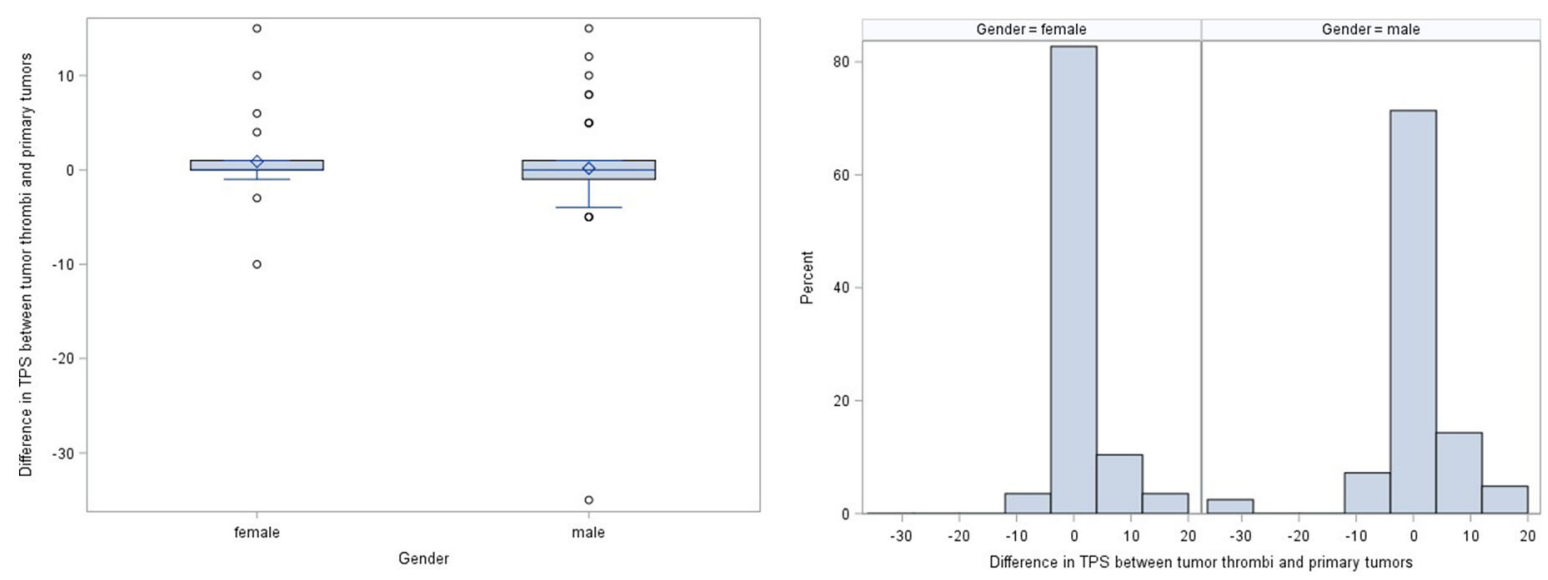

Fig. 12 Box plots and histograms of the difference in TPS between venous tumor thrombi and primary tumors according to gender. TPS Tumor Proportion Score

even complete responses. Despite their oncologic benefits, they have not been widely recommended because of their toxicity and low levels of efficacy. Vascular endothelial growth factor (VEGF)-targeted agents, which include TKIs in addition to mammalian target of rapamycin (mTOR) inhibitors, have been introduced as potential targeted therapies with improved survival rates. They have been mentioned as milestones in the initiation of targeted molecular therapies $[4,6]$. The introduction of ICIs has changed the therapeutic strategies used for metastatic renal cell carcinoma (mRCC). ICIs have fewer side effects, better HRQoL and improved outcomes with, in some cases, even complete responses $[6,7]$. Despite producing some intriguing results, they are not always beneficial to every patient [8]. Nowadays, miscellaneous immune checkpoint inhibitor agents can be used alone or in combination with other types of mRCC therapies, including TKIs and other ICIs.

Studies have shown that PD-L1 expression in tumors can vary depending on the lesion [8]. In contrast, Callea et al.'s study demonstrated that there are no significant differences between the PD-L1 expression characteristics of primary RCC and paired metastases. Interestingly, they found that there were significant variations between clinical influencing factors in the expression of PD-L1 within PTs [13]. In our study, the analysis of PD-L1 expression patterns, as measured by the CPS, IC score and TPS, showed that no statistically significant differences existed between PTs and VTT in paired samples. Furthermore, regarding the possible influencing factors of gender and age at the time of the initial surgery, the variation in PD-L1 expression between the VTT and PTs was not statistically different.

All the main phase 3 immune checkpoint inhibitor trials involving patients with mRCC (Checkmate 214, Keynote 426, Javelin RENAL 101 and IMmotion 151) used one of the aforementioned PD-L1 scoring systems to investigate the PT [2]. PD-L1 determinations in the metastases and a comparison between the metastases and the PT were not considered. In all these trials, the cutoff value for PD-L1 positivity was defined as $\geq 1 \%$ [2]. Knowing that ICIs are nonresponsive in the case of PD-L1-positive RCCs, but produce positive responses in some PD-L1-negative tumors [13], we cannot define any decisive role for this biomarker, unaided, in treatment strategies. The KEYNOTE-059 study demonstrated the importance of assessing PDL1 expression through a number of simultaneous methods. This study highlighted that PD-L1 expression can be better detected by using the CPS instead of the TPS in refractory and metastatic gastric or gastroesophageal cancers [12]. Accordingly, we evaluated the CPS, IC and TPS modalities in our aRCC with VTT patients to achieve a better understanding of them. 
There are also limited data on the therapy options available to aRCC with VTT patients [14]. If the best prognostic treatment is considered to be the removal of the VTT, particularly when the vena cava is involved, more perioperative complications can result regardless of the surgical method used. This option can be particularly life-threatening in older patients who may have other underlying health conditions, and this makes the surgical treatment of aRCC with VTT more challenging. In these cases, knowledge of the PD-L1 status in the VTT is invaluable. Downstaging of the VTT through neoadjuvant treatment with ICIs can play an important role in minimizing surgical complications. The positive impact of the use of ICIs as neoadjuvant therapies in aRCC and mRCC cases with VTT has been reported $[15,16]$.

It is crucial not to forget that the use of ICIs in mRCC is a palliative treatment, and immunerelated adverse events (irAEs) are warranted. With better oncologic results compared to medication alone, combination therapies should not cause excessive irAEs. According to current studies, the majority of irAEs are less dangerous and more conservatively manageable than the adverse-event profiles of previous therapies. As long as any irAEs are quickly diagnosed, their management is a feasible option. Again, this highlights the necessity of an appropriate biomarker to control the optimal levels of ICIs, their effectiveness and the early detection of irAEs.

As stated in the current recommendations of the European Association of Urology (EAU) 2020 , the combination of pembrolizumab (as one of the ICIs) and axitinib (as one of the TKIs) is now an established front-line therapy for patients with clear-cell mRCC in all risk groups, who have not undergone any previous treatment, according to the International Metastatic Renal Cell Carcinoma Database Consortium (IMDC). ICIs have always played a central role in metastatic settings. We are concerned with the role played by ICIs in downstaging the nonmetastatic, locally aRCC scenarios, including their therapeutic (not palliative) significance. Nonetheless, according to the EAU Guidelines 2020, the PD-L1 biomarker status (in PTs) is currently not recommended as a decisive measurement by which the treatment of aRCC and mRCC patients can be determined.

The role of VEGF expression in the molecular pathogenesis of RCC and comparisons between vascular endothelial growth factor receptor 1 (VEGFR1) and vascular endothelial growth factor ligand D (VEGFD) in PTs, VTT and mRCC have previously been published by a number of authors. VEGFR1 expression, as a known prognosticator, showed a meaningful increase in metastases compared to PTs or VTT, without a significant difference being observed between PTs and VTT [17]. Considering that ICIs are recommended as a first-line treatment in combination with TKIs, further comprehensive studies are needed to understand the roles and effects of each medication in the aforementioned combination.

The soluble plasma PD-L1 protein, as an independent prognosticator in ccRCC, is a good example of a newly published proposed method in classifying and controlling disease apart from intratumor heterogeneity (ITH) causing variable PD-L1 expression in tumor tissues [18]. ITH influences diagnosis, treatment and follow-up, which might mislead management of patients [8, 19]. A multisite tumor sampling strategy (MSTS) has been shown to be superior in correct determination of PD-L1 expression [20]. In our series, original sampling was performed as recommended by the respective societies, i.e., one sample per $\mathrm{cm}$ of tumor size and inclusion of the invasive front as well as the border of necrosis. For PD-L1 scoring, samples selected from paraffin archives included the worst differentiation of the tumor and margin of necrosis, if possible. However, heterogeneity was still evident and might be a confounder of our results $[19,20]$.

This work has additional limitations. The data were obtained from a single clinic and evaluated retrospectively. In contrast to our study, Lopez et al. compared 39 aRCC cases and described a significant decrease of PD-L1 expression in VTT compared to PTs [21]. The discrepant result compared to our study (stainings with 22C3) might be explained by the use of a different antibody (SP-142). Further studies with a prospective design and larger cohorts are recommended. If the results of the current 
study can be confirmed in larger study groups, this could lead to new therapeutic approaches in the treatment of aRCC with VTT.

Even though there are controversies and the aforementioned unexplained facts surrounding the use of ICIs, they are still considered to be a worthwhile state-of-the-art therapy for mRCC. Therefore, it makes sense to find an appropriate predictor to assess treatment responses and to consider how therapies can be combined to produce a treatment with the best tolerance levels and the least adverse effects in aRCC. The importance and correlation of the expression of different VEGF and PD-L1 modalities are part of the next stage in the advancement of molecular biomarkers, which could enable a personalized aRCC therapy to be developed even as a curative means.

\section{CONCLUSION}

Our study has shown that similar PD-L1 expression patterns are found in the primary tumors and venous tumor thrombi of advanced kidney cancer. We hope that our findings will serve as a potential neoadjuvant therapy for aRCC with VTT. The neoadjuvant treatment of aRCC with VTT by using ICIs in combination with TKIs for downstaging, particularly in those cases where the vena cava is involved, could be an alternative and less aggressive curative option than primary surgery. The establishment of immune checkpoint inhibitors in advanced kidney cancer $\geq$ pT3a requires further comprehensive molecular research and clinical trials.

\section{ACKNOWLEDGEMENTS}

We would like to thank Edda Christians and Mahtab Taleb-Naghsh for their excellent technical assistance.

Funding. Open Access funding enabled and organized by Projekt DEAL. We would like to express our appreciation to the Wehrmann Foundation and Wilhelm Sander Foundation (JHB, JS) for their financial support. No Rapid
Service Fee was received by the journal for the publication of this article.

Authorship. All named authors meet the International Committee of Medical Journal Editors (ICMJE) criteria for authorship for this article, take responsibility for the integrity of the work as a whole, and have given their approval for this version to be published.

Authorship Contributions. MM contributed to manuscript writing/editing, data analysis and protocol/project administration. RR contributed to manuscript writing/editing, data analysis and data collection/management. PFT contributed to data collection/management, review and editing. MA contributed to conceptualization, methodology and data analysis. MW contributed to protocol/project development, and editing. JS contributed to data collection/management and data curation. JHB contributed to data collection/management, review and editing. IP contributed to investigation, methodology and writing original draft. MAK contributed to concept/design and protocol/project development (consultant). HT was the project leader and contributed to protocol/ project development, manuscript writing/editing, data analysis and data collection/management.

Disclosures. Mehrdad Mazdak, Rieke Ringlstetter, Pouriya Faraj Tabrizi, Meryem Akkoyun, Mathias Wolters, Jessica Schmitz, Jan Hinrich Bräsen, Inga Peters, Markus Antonius Kuczyk and Hossein Tezval have nothing to disclose.

Compliance with Ethics Guidelines. The study was approved by the ethical committee of the Hanover Medical School (MHH) (ethics votes number 1696-2013). All procedures performed in our study were in accordance with the ethical standards of the institutional research committee and with the 1964 Helsinki Declaration and its later amendments or comparable ethical standards.

Data Availability. The datasets generated during and/or analyzed during the current 
study are available from the corresponding author on reasonable request.

No Rapid Service Fee was received by the journal for the publication of this article.

Open Access. This article is licensed under a Creative Commons Attribution-NonCommercial 4.0 International License, which permits any non-commercial use, sharing, adaptation, distribution and reproduction in any medium or format, as long as you give appropriate credit to the original author(s) and the source, provide a link to the Creative Commons licence, and indicate if changes were made. The images or other third party material in this article are included in the article's Creative Commons licence, unless indicated otherwise in a credit line to the material. If material is not included in the article's Creative Commons licence and your intended use is not permitted by statutory regulation or exceeds the permitted use, you will need to obtain permission directly from the copyright holder. To view a copy of this licence, visit http://creativecommons.org/licenses/by$\mathrm{nc} / 4.0 /$.

\section{REFERENCES}

1. Linehan WM, Ricketts CJ. The metabolic basis of kidney cancer. Semin Cancer Biol. 2013;23(1): 46-55.

2. Garje R, et al. The future of immunotherapy-based combination therapy in metastatic renal cell carcinoma. Cancers (Basel). 2020;12(1):143.

3. Wotkowicz C, Wszolek MF, Libertino JA. Resection of renal tumors invading the vena cava. Urol Clin North Am. 2008;35(4):657-71.

4. Alsharedi M, Katz H. Check point inhibitors a new era in renal cell carcinoma treatment. Med Oncol. 2018;35(6):85.

5. Zhang X, et al. Differential expressions of PD-1, PDL1 and PD-L2 between primary and metastatic sites in renal cell carcinoma. BMC Cancer. 2019;19(1): 360 .

6. Cetin B, Kosar A. Game of thrones: immunotherapy versus molecular targeted therapy in renal cell cancer scenarios. Int Urol Nephrol. 2019;51(12): 2107-17.
7. Cella D, et al. Patient-reported outcomes of patients with advanced renal cell carcinoma treated with nivolumab plus ipilimumab versus sunitinib (CheckMate 214): a randomised, phase 3 trial. Lancet Oncol. 2019;20(2):297-310.

8. Nunes-Xavier CE, et al. A critical insight into the clinical translation of PD-1/PD-L1 blockade therapy in clear cell renal cell carcinoma. Curr Urol Rep. 2019;20(1):1

9. Huang JJ, Hsieh JJ. The therapeutic landscape of renal cell carcinoma: from the dark age to the golden age. Semin Nephrol. 2020;40(1):28-41.

10. Schildhaus HU. Predictive value of PD-L1 diagnostics. Pathologe. 2018;39(6):498-519.

11. Trpkov $\mathrm{K}$, et al. Handling and staging of renal cell carcinoma: the International Society of Urological Pathology Consensus (ISUP) conference recommendations. Am J Surg Pathol. 2013;37(10): 1505-17.

12. Kulangara $\mathrm{K}$, et al. Clinical utility of the combined positive score for programmed death ligand-1 expression and the approval of pembrolizumab for treatment of gastric cancer. Arch Pathol Lab Med. 2019;143(3):330-7.

13. Callea $\mathrm{M}$, et al. Differential expression of PD-L1 between primary and metastatic sites in clear-cell renal cell carcinoma. Cancer Immunol Res. 2015;3(10):1158-64.

14. Psutka SP, Leibovich BC. Management of inferior vena cava tumor thrombus in locally advanced renal cell carcinoma. Ther Adv Urol. 2015;7(4): 216-29.

15. Labbate $\mathrm{C}$, et al. Complete response of renal cell carcinoma vena cava tumor thrombus to neoadjuvant immunotherapy. J Immunother Cancer. 2019;7(1):66.

16. Berends J, Gourley E, Kaushik D. Robust response to nivolumab in patient with renal cell carcinoma inferior vena cava tumour thrombus. BMJ Case Rep. 2019;12(4):e227030.

17. Laird A, et al. Differential expression of prognostic proteomic markers in primary tumour, venous tumour thrombus and metastatic renal cell cancer tissue and correlation with patient outcome. PLoS One. 2013;8(4):e60483.

18. Larrinaga G, et al. Soluble PD-L1 is an independent prognostic factor in clear cell renal cell carcinoma. Cancers (Basel). 2021;13(4):667. 
19. Lopez JI, Angulo JC. Pathological bases and clinical impact of intratumor heterogeneity in clear cell renal cell carcinoma. Curr Urol Rep. 2018;19(1):3.

20. Lopez JI, et al. Potential impact of PD-L1 (SP-142) immunohistochemical heterogeneity in clear cell renal cell carcinoma immunotherapy. Pathol Res Pract. 2018;214(8):1110-4.

21. Lopez JI, et al. Loss of PD-L1 (SP-142) expression characterizes renal vein tumor thrombus microenvironment in clear cell renal cell carcinoma. Ann Diagn Pathol. 2018;34:89-93. 